\title{
Eg5 high expression predicts dismal prognosis in epithelial ovarian cancer
}

\author{
Pingping Sun ${ }^{1}$, Xuemin $\mathrm{Cao}^{2}$, Yuanlin Liu ${ }^{3}$, Lizhou Jia ${ }^{4 *}$ \\ ${ }^{1}$ Department of Clinical Biobank, Affiliated Hospital of Nantong University, Nantong, Jiangsu 226001, China; \\ ${ }^{2}$ Key Laboratory of Neuroregeneration, Nantong University, Nantong, Jiangsu 226001, China; \\ ${ }^{3}$ Department of Obstetrics \& Gynecology, Affiliated Hospital of Nantong University, Nantong, Jiangsu 226001, China; \\ ${ }^{4}$ Department of Surgical Oncology, Bayannur Hospital, Bayannur, Inner Mongolia 015000, China.
}

\begin{abstract}
The expression of kinesin spindle protein (Eg5) and its significance of clinical prognosis of patients with epithelial ovarian cancer (EOC) were evaluated in this study. Forty-five fresh frozen tissue samples for quantitative real-time polymerase chain reaction (qPCR) and 196 samples for immunohistochemistry (IHC) analysis with tissue microarray (TMA) were applied to characterize Eg5 mRNA and protein expressions in EOC. The correlation between clinical parameters and Eg5 protein expression was investigated using statistical analysis. The expression of Eg5 protein was significantly higher in EOC tissues compared with that in corresponding non-cancerous tissues $(P<0.05)$. The high Eg5 expression was significantly correlated with older age $(P=0.003)$, higher stage $(P=$ $0.001)$, presence of metastasis $(P=0.041)$ and higher CA125 serum level $(P=0.013)$. For univariate analysis, associated prognostic markers in patients with ovarian cancer were analyzed for correlations with poor overall survival, including Eg5 $(P=0.011)$, age $(P=0.001)$, FIGO stage $(P=0.011)$, CA125 serum level $(P=0.001)$, lymph nodes $(P=0.012)$, and metastasis $(P=0.001)$. For multivariate analysis, Eg5 expression, FIGO stage and age were independent factors found contributing to a largely unfavorable prognosis in patients with ovarian cancer. In conclusion, the high expression of Eg5 is correlated with an unfavorable prognosis in EOC.
\end{abstract}

Keywords: Eg5; ovarian cancer; qPCR; IHC; prognosis

\section{INTRODUCTION}

Epithelial ovarian cancer (EOC) is a high malignancy gynecological cancer with a 5-year average survival rate of $30 \%{ }^{[1]}$. Worldwide, the number of EOC cases has shown an increasing trend. Most patients cannot be diagnosed until they are at an advanced stage ${ }^{[2-3]}$. Although progress has been made in diagnosis and treatment, the majority of patients with EOC are resistant to chemotherapy, and their prognosis still remains poor ${ }^{[4-5]}$. In recent years, the prognosis of EOC patients has mostly depended on the completeness of surgical tumor resection, and no established preoperative markers have been found to predict EOC patients' overall survival.

Kinesin spindle protein(Eg5), also known as KIFl1, $\mathrm{KSP}, \mathrm{KNSL1}$, is a motor protein which catalyzes the bipolarization of the spindle, and is responsible for spindle elongation and poleward MT translocation ${ }^{[6-7]}$. Eg5 can tether microtubules' plus-ends or push apart

*Correspondence to: Lizhou Jia, Department of Surgical Oncology, Bayannur Hospital, 98 Wulanbuhe Road, Bayannur, Inner Mongolia 015000, China. E-mail: nmgjlz@163.com

Conflict of interests: The authors have no conflicts of interest. 
interpolar microtubules, as well as recruit microtubules into bundles ${ }^{[8]}$. Structurally, Eg5 is a homotetramer, which includes four kinetic domains: two polypeptide chains are dimerized to form a coiled-coil, while two dimers form an anti-parallel tetramer helix structure $^{[9]}$. Recent studies have evaluated the significance of Eg5 expression in different kinds of malignant tumors ${ }^{[10-11]}$. For example, the overexpression of Eg5 in malignant astrocytic neoplasms, has been found to correlate significantly to their histopathological grade ${ }^{[12]}$. The overexpression of Eg5 has also been found to interfere with the balance of normal spindle assembly and function-related forces, leading to spindle defects, genetic instability and tumor development ${ }^{[13]}$. Eg5 inhibitors, as targeted drugs have positive effects in the treatment of prostate cancer in vivo where it is believed that the inhibition of Eg5 leads to the stagnation of the mitotic cell cycle, ultimately triggering growth arrest and apoptosis in tumor cells ${ }^{[14-16]}$. These studies indicate that the oncogenicity of Eg5 forms an attractive target for the chemotherapeutic management of cancer. However, the potential association between Eg5 expression and its clinicopathological significance in EOC has not been studied.

Here, Eg 5 mRNA expression was detected using one-step quantitative reverse transcription-polymerase chain reaction (qPCR) analysis, while Eg 5 protein expression was measured by immunohistochemistry (IHC) analysis. The relationship between Eg5 expression and EOC clinicopathology, as well as patient prognostic outcomes including overall survival and disease-free survival were evaluated.

\section{MATERIALS AND METHODS}

\section{Patient specimens}

This study samples were collected from the Department of Gynecology, Affiliated Hospital of Nantong University from July 2005 to July 2010. Total 196 samples included 15 cases with nor-mal ovarian tissue, 30 cases of normal fallopian tube tissue, 20 cases of ovarian benign tumor, 20 cases of borderline ovarian tumor and 111 cases of EOC. The 15 cases with normal ovarian tissue of control group were removed from patients with non-ovarian disease. Those 111 cases of EOC included 84 cases of serous carcinoma, 13 cases of endometrioid carcinoma, 4 cases of clear cell carcinoma, 5 cases of mucinous carcinoma, 2 cases of transitional cell carcinoma, and 3 cases of adeno-squamous carcinoma.

All patients underwent standard surgery without any therapy prior to surgery, such as chemotherapy, radiation or immunotherapy. Then, all EOC patients were treated with platinum-based chemotherapy at least six times after surgery. All samples gained medical-ethics approval from the Nantong University Affiliated Hospital's Human Research Ethics Committee.

\section{Eg5 mRNA isolation and real-time qPCR assay}

There were 45 fresh frozen tissue samples, including 15 cases of normal ovarian tissue, 20 cases of benign ovarian tumor and 10 cases of EOC. Total RNA was extracted using Trizol reagent (Invitrogen, Carlsbad, CA) according to instructions. qPCR was performed using SyberGreen on an ABI 7500 Thermal Cycler (Applied Biosystems, Foster City, CA, USA). The qPCR conditions were as fol-lows: UDG pre-treatment at $50^{\circ} \mathrm{C}$ for 2 min, 1 cycle; initial denaturation at $95^{\circ} \mathrm{C}$ for $10 \mathrm{~min}$; denaturation at $95^{\circ} \mathrm{C}$ for $15 \mathrm{~s}$; and annealing and extension at $60^{\circ} \mathrm{C}$ for $60 \mathrm{~s}, 40$ cycles. The primers for Eg5 were as follows: forward primer 5'-GAA CAA TCA TTA GCA GCA GAA$3^{\prime}$ and reverse primer 5'-TCA GTA TAG ACA CCA CAG TTG-3'. $\beta$-actin was employed as an internal control: forward primer $5^{\prime}$-TAA TCT TCG CCT TAA TAC TT -3 ' and reverse primer $5^{\prime}-\mathrm{AGC}$ CTT CAT ACA TCT CAA-3'.

\section{Immunohistochemistry (IHC) analysis}

IHC analysis was performed as described previously ${ }^{[16-17]}$. Antigen repair was executed in citrate buffer ( $\mathrm{pH}$ 6.0) under microwave heating conditions. TMA sections were incubated with $3 \% \mathrm{H}_{2} \mathrm{O}_{2}$ for 20 min to remove endogenous enzymes, then incu-bated with $5 \%$ bovine serum albumin (BSA) in PBS for 20 min for blocking non-specific binding. After washing with phosphate buffer, a polyclonal anti-Eg 5 antibody ( $1: 100$, Abcam, Cambridge, USA) was applied to the sections overnight at $4{ }^{\circ} \mathrm{C}$. The next day, samples were incubated with Envision goat anti-rabbit HRP secondary antibody (DAKO, Carpinteria, CA). IHC experiment methods were performed at the same time under the same conditions. Immunostained sections were observed through a common upright microscope and scored by two independent pathologists under blind experimental conditions according to intensity and percentage of Eg5-positive cells.

The intensity of Eg5-positive cells was scored as follows: 0 (no intensity), 1 (mild intensity), 2 (medium intensity), or 3 (strong intensity). The percentage of Eg5-positive cells was scored as follows: 0 for $0 \%-19 \%, 1$ for $20 \%-39 \%, 2$ for $40 \%-59 \%$ and 3 for $60 \%-100 \%$. The optimal Eg5 cutoff score was set using the X-tile software program (The Rimm Lab at Yale University; http://www.tissuearray.org/rimmlab). The final Eg5 staining score (including the intensity 
and percentage scores) was defined as follows: 0-4 for no or low expression; 6-9 for high expression.

\section{Statistical analysis}

Eg5 mRNA expression in fresh EOC tissues was compared with that of corresponding non-cancerous tissues by the Wilcoxon signed rank nonparametric test. A chi-square test was conducted to examine the association of Eg5 protein expression and clinicopathological parameters. Kaplan-Meier and log-rank tests were performed to calculate the survival curves. The univariate and multivariate analyses were identified using a Cox regression model for the prognostic factors. For all tests, the statistical significance level was set at $P<0.05$. All statistics were analyzed using STATA Version 12.0 software (Stata Corporation, College Station, TX).

\section{RESULTS}

\section{Eg5 mRNA expression in ovarian tissues}

To test the expression of Eg5 mRNA, qPCR was performed for normal ovary, benign ovarian tumor and EOC samples. Eg5 mRNA expression was significantly higher in EOC tissues $(0.7489 \pm 0.0421)$ compared with those of benign ovarian tumor tissues $(0.2645 \pm 0.0253)$ and normal ovarian tissues $(0.2851$ $\pm 0.0226)(P<0.001$, Fig.1 $)$.

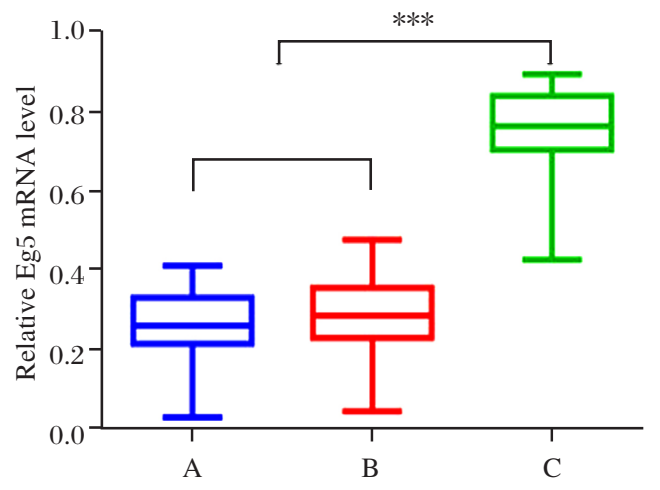

Fig.1 Eg5 mRNA level was higher in EOC tissues than those in normal and benign ovarian tissues. $\mathrm{A}$ : Normal ovary samples. B: Benign ovarian tumor samples. C: Epithelial ovarian cancer samples. Eg5 mRNA was detected by real-time RT-PCR assay in normal ovary, benign ovarian tumor and epithelial ovarian cancer samples, followed by relative quantitative analysis with $\beta$-actin mRNA. Data are presented as means \pm SD. ${ }^{* * *} P<0.001$.

\section{Eg5 protein expression in ovarian tissues}

For further investigation, TMA immunohistochemistry analysis was performed to evaluate $\mathrm{Eg} 5$ protein expression in ovarian tissue blocks (196 in total), including 15 normal ovarian tissues, 30 normal fallopian tube tissues, 20 benign ovarian tumor tissues, 20 borderline ovarian tumor tissues and 111 EOC tissues. Eg5 positive staining was mainly detected in the cytoplasm of ovarian cancer cells (Fig.2), which is consistent with previous Eg 5 mRNA detection results. Eg5 high expression was detected in $61.26 \%(68 / 111)$ of EOC cases, and in $13.33 \%-25.00 \%$ of normal or benign ovarian tissues. The results showed that the frequency of high Eg5 expression in EOC tissues was higher compared to those of normal or benign ovarian tissues (Pearson $\chi^{2}=45.486, P=0.001$, Table 1).

\section{Association between Eg5 expression and EOC clinical characteristics}

Furthermore, we correlated Eg5 expression with

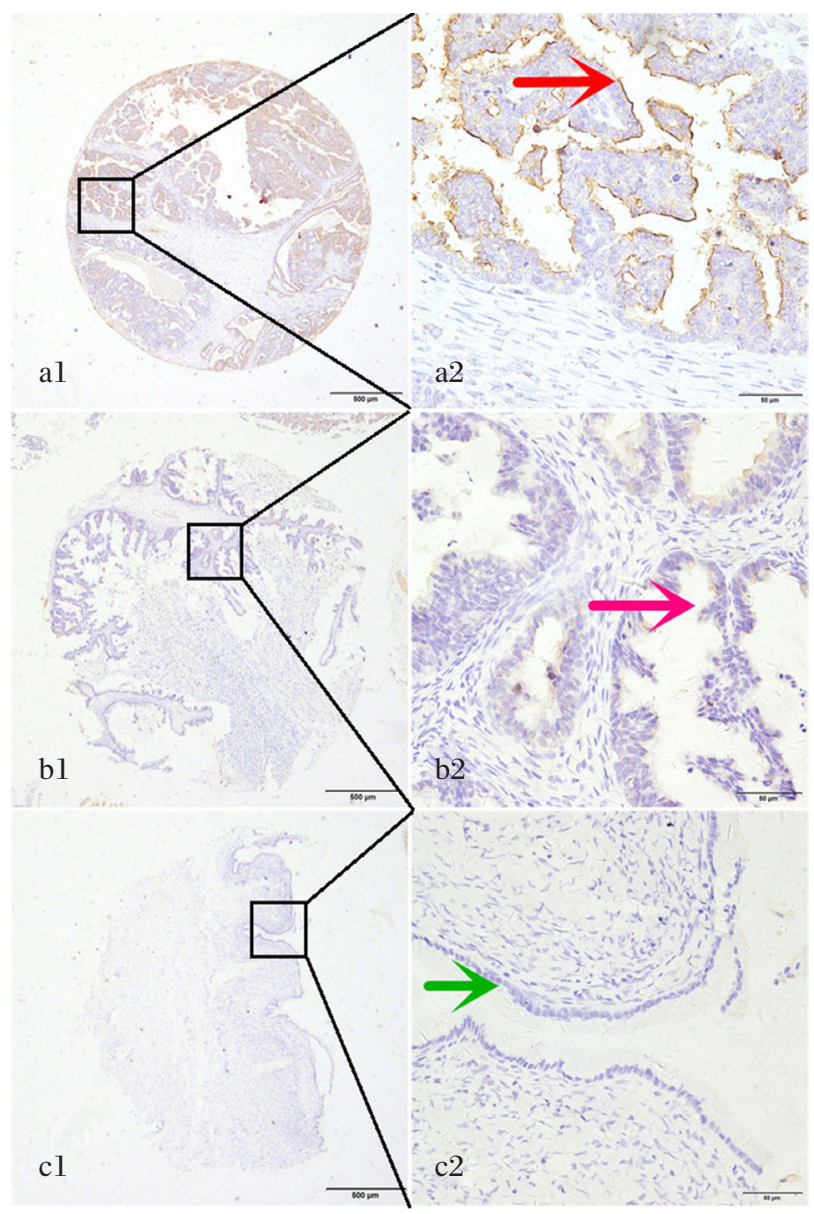

Fig.2 Eg5 Immunohistochemical analysis in ovarian cancer tissues and normal ovarian tissues. ala2: Strong positive for Eg5 protein expression in EOC tissues. b1-b2: Weakly positive for Eg5 protein expression in benign ovarian tumor tissues. c1-c2: Negative for Eg5 protein expression in normal ovarian tissues. al, bl and cl were $\times 40$ magnifification $(\mathrm{bar}=500 \mu \mathrm{m})$; $\mathrm{a} 2, \mathrm{~b} 2$ and $\mathrm{c} 2$ were $\times 200$ magnifification $($ bar $=50 \mu \mathrm{m})$. Red arrow indicates positive staining of cytoplasm in ovarian cancer cells, and green arrow indicates negative staining of follicular epithelium in ovarian tissue. 
Table 1 Eg5 expressions in normal ovarian, normal fallopian tube, benign ovarian tumor, borderline ovarian tumor and EOC tissues

\begin{tabular}{|c|c|c|c|c|c|}
\hline \multirow{2}{*}{ Characteristic } & \multirow{2}{*}{$\begin{array}{c} \\
(196)\end{array}$} & \multicolumn{4}{|c|}{ Eg5 expression } \\
\hline & & None or low & High & Pearson $\chi^{2}$ & $P$-value \\
\hline & & & & 45.486 & $0.001^{*}$ \\
\hline Normal ovarian tissue & 15 & $12(80.00)$ & $3(20.00)$ & & \\
\hline Normal fallopian tube tissue & 30 & $26(86.67)$ & $4(13.33)$ & & \\
\hline Benign ovarian tumor & 20 & $17(85.00)$ & $3(15.00)$ & & \\
\hline Borderline ovarian tumor & 20 & $15(75.00)$ & $5(25.00)$ & & \\
\hline Epithelial ovarian cancer & 111 & $40(36.04)$ & $71(63.96)$ & & \\
\hline
\end{tabular}

111 EOC patients who had clinical characteristics. Among them, $57.66 \%$ were grouped as younger $(\leqslant$ 60 years, $n=64)$ and $42.34 \%$ patients older $(>60$ years, $n=47)$. With respect to tumor grade, $22 \mathrm{pa}-$ tients $(19.82 \%)$ were classified as low grade, while 89 patients $(80.18 \%)$ were high grade. In addition, 84 patients $(57.68 \%)$ presented with serious carcinoma, 13 patients $(11.71 \%)$ with endometrioid carcinoma and 14 patients $(12.61 \%)$ with other carcinomas. The FIGO stage distribution was 41 patients $(36.94 \%)$ with lower stage (FIGO stage I-II) and 70 patients $(63.06 \%)$ with higher stage (FIGO stage III-IV). Regarding tumor metastasis, 55 patients (49.55\%) had metastases, while the remaining patients did not. For tumor sites, 66 patients $(59.46 \%)$ were unilateral and $45 \mathrm{pa}-$ tients $(40.54 \%)$ were bilateral. Finally, 44 patients $(39.64 \%)$ had lower CAl2 25 values $(\leqslant 100 \mathrm{U} / \mathrm{mL})$ and 67 patients $(60.36 \%)$ had higher CAl 25 values
$(>100 \mathrm{U} / \mathrm{mL})$.

The chi-square test analysis showed that the Eg5 expression was significantly correlated with older age ( $>60$ years, $P=0.003$ ), higher stage (FIGO stage III$\mathrm{IV}, \mathrm{P}=0.001)$, presence of metastasis $(P=0.041)$ and higher serum CA125 level (> $100 \mathrm{U} / \mathrm{mL}, P=0.013)$. In contrast, high Eg5 expression was not significantly correlated with other clinical indicators, including tumor grade, histological classification and tumor site (Table 2).

\section{High Eg5 protein expression predicts poor survival in EOC}

Both univariate and multivariate analysis were used to identify prognostic factors in EOC. For univariate analyses, associated prognostic markers were analyzed for correlations with poor overall survival,

Table 2 Correlation of Eg5 expression with clinicopathological characteristics in ovarian cancer $[n(\%)]$

\begin{tabular}{|c|c|c|c|c|c|}
\hline \multirow{2}{*}{ Groups } & \multicolumn{5}{|c|}{ Eg5 } \\
\hline & $N$ & None or low & High & Pearson $\chi^{2}$ & $P$ \\
\hline Age & & & & 8.779 & $0.003^{*}$ \\
\hline$>60$ years & 47 & $16(34.04)$ & $31(66.96)$ & & \\
\hline Grade & & & & 0.001 & 0.971 \\
\hline Low & 22 & $8(36.36)$ & $14(63.64)$ & & \\
\hline High & 89 & $32(35.96)$ & $57(64.04)$ & & \\
\hline Histological classification & & & & 1.126 & 0.569 \\
\hline Serous carcinoma & 84 & $28(33.33)$ & $56(66.67)$ & & \\
\hline Endometrioid carcinoma & 13 & $6(46.15)$ & $5(53.85)$ & & \\
\hline Othera & 14 & $6(42.86)$ & $8(57.14)$ & & \\
\hline FIGOb stage & & & & 29.384 & $0.001^{*}$ \\
\hline $\mathrm{I}-\mathrm{II}$ & 41 & $28(68.29)$ & $13(31.71)$ & & \\
\hline Metastasis & & & & 4.196 & $0.041^{*}$ \\
\hline Yes & 55 & $25(45.45)$ & $30(54.54)$ & & \\
\hline No & 56 & $15(26.79)$ & $41(73.21)$ & & \\
\hline Unilateral or bilateral & & & & 0.100 & 0.752 \\
\hline unilateral & 66 & $23(34.85)$ & $43(65.15)$ & & \\
\hline bilateral & 45 & $17(37.78)$ & $28(62.22)$ & & \\
\hline CA125 & & & & 6.167 & $0.013^{*}$ \\
\hline$\leqslant 100$ & 44 & $22(50.00)$ & $22(50.00)$ & & \\
\hline$>100$ & 67 & $18(26.87)$ & $49(73.13)$ & & \\
\hline
\end{tabular}

${ }^{*} P<0.05$.

${ }^{\text {a }}$ Clear cell carcinoma, 4 cases; mucinous carcinoma, 5 cases; transitional cell carcinoma, 2 cases; adeno-squamous carcinoma, 3 cases.

${ }^{\mathrm{b}}$ FIGO: Federation of Gynecologists and Obstetrics 
including: Eg5 (HR: 2.111, 95\% CI: 1.188-3.752, $P$ $=0.011)$, age (HR: $2.398,95 \%$ CI: $1.431-4.019, P=$ 0.001 ), FIGO stage (HR: $2.113,95 \%$ CI: 1.190-3.752, $P=0.011$ ), CA125 level in serum (HR: $4.321,95 \%$ CI: 2.306-8.098, $P=0.001$ ), lymph nodes (HR: 2.114, 95\% CI: 1.184-3.875, $P=0.012$ ), and metastasis (HR: $4.748,95 \%$ CI: $2.651-8.507, P=0.001)$. For mul- tivariate analyses, high Eg5 expression (HR: 1.915, 95\% CI: $1.115-3.289, P=0.019)$ and high CAl25 serum level (HR: 3.107, 95\% CI: 1.594-6.059, $P=$ 0.001 ) were independent prognostic factors correlated with poor overall survival (Table 3). The KaplanMeier survival curve showed similar results to the multivariate analysis $(\log$ rank, $P<0.001$, Fig.3).

Table 3 Univariate and multivariate Cox proportional hazard model analysis of prognostic markers for overall survival in ovarian cancer

\begin{tabular}{|c|c|c|c|c|c|c|c|}
\hline \multirow{2}{*}{ Groups } & \multicolumn{5}{|c|}{ Eg5 } & \multirow[b]{2}{*}{$P$} & \\
\hline & $N$ & None or low & High & & Pearson $\chi^{2}$ & & \\
\hline \multicolumn{8}{|l|}{ Eg5 expression } \\
\hline Low vs high & 2.111 & $0.011^{*}$ & $1.188-3.752$ & 1.915 & $0.019^{*}$ & & $1.115-3.289$ \\
\hline \multicolumn{8}{|l|}{ Age (years) } \\
\hline$\leqslant 60 v s>60$ & 2.398 & $0.001^{*}$ & $1.431-4.019$ & & & & \\
\hline \multicolumn{8}{|l|}{ Grade } \\
\hline \multicolumn{8}{|l|}{ Pathological type } \\
\hline Unilateral vs bilateral & 1.463 & 0.144 & $0.878-2.437$ & & & & \\
\hline \multicolumn{8}{|l|}{ CA125 } \\
\hline Low vs high & 4.321 & $0.001^{*}$ & $2.306-8.098$ & 3.107 & $0.001^{*}$ & & $1.594-6.059$ \\
\hline \multicolumn{8}{|l|}{ Lymph nodes } \\
\hline None vs yes & 2.114 & $0.012^{*}$ & $1.184-3.875$ & & & & \\
\hline None vs yes & 4.748 & $0.001^{*}$ & $2.651-8.507$ & & & & \\
\hline \multicolumn{8}{|l|}{ Type } \\
\hline Serous vs others & 0.875 & 0.511 & $0.587-1.304$ & & & & \\
\hline \multicolumn{8}{|l|}{ FIGO stage } \\
\hline Stage I vs stage II-IV & 2.113 & $0.011^{*}$ & $1.190-3.752$ & & & & \\
\hline
\end{tabular}

vs: versus

HR: Hazard ratio; CI: Confidence interval. ${ }^{*} P<0.05$.

FIGO: Federation of Gynecologists and Obstetrics
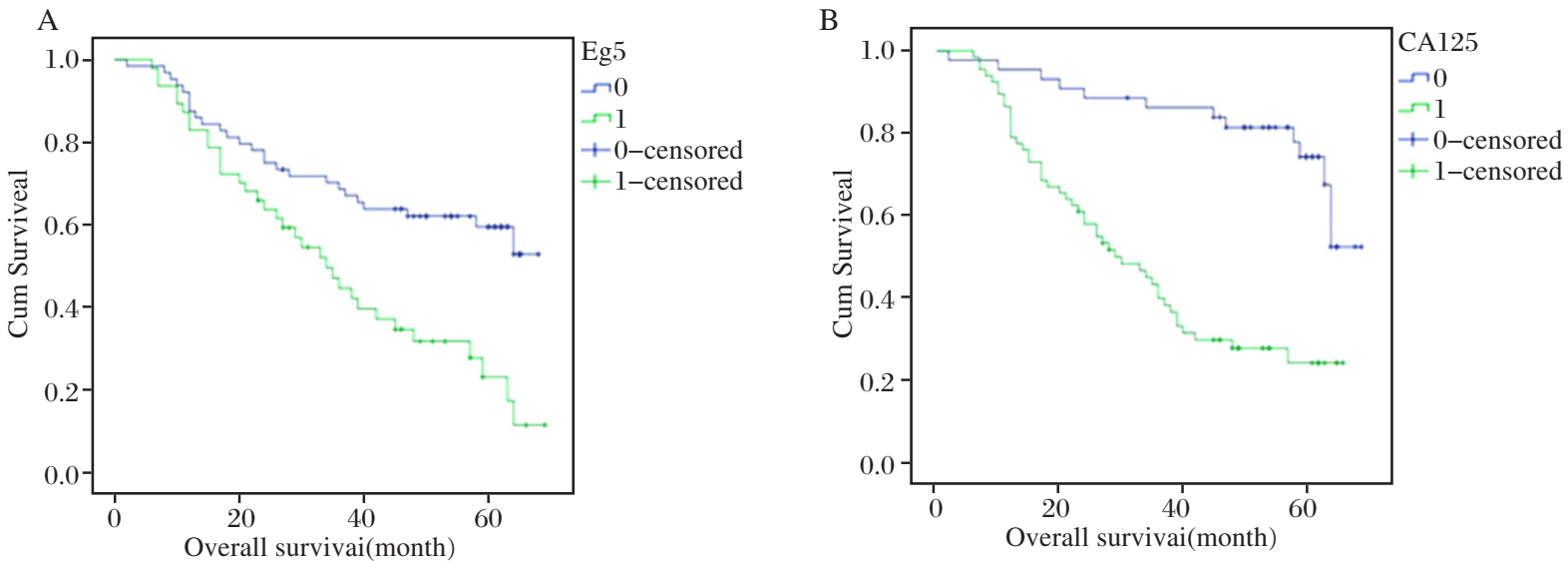

Fig.3 Survival curves of EOC patients according to Eg5 expression and CA125 level by Kaplan-Meier method. A: Overall survival curves of high Eg5 expression (green line, 1) and low Eg5 expression (blue line, 0) in EOC patients. B: Overall survival curves of high CA125 level (green line, 1) and low CA125 level (blue line, 0) in EOC patients.

\section{DISCUSSION}

In this study, the expression of Eg5 in ovarian cancer specimens and non-cancerous samples were compared. The results indicated that Eg5 mRNA expression and protein levels were significantly higher in EOC than those in normal and benign ovarian tissues. In the IHC analysis, the Eg5 expression pattern was predominantly localized to the EOC cell cytoplasm. In addition, this study also found that high Eg5 protein expression was associated with the following clinicopathological attributes: older age, higher stage, 
presence of metastasis and higher CA125 serum level. This finding suggests that the expression of $\mathrm{Eg} 5$ is related to malignant behaviors in the development of EOC.

Eg5, a member of the kinesin family, is involved in spindle formation and tumorigenesis ${ }^{[17]}$. Hayashi et al. ${ }^{[18]}$.reported that Eg5 expression levels seem to be directly proportional to the mitotic population in prostate cell lines Marcus et al. ${ }^{[19]}$ stated that there was a positive correlation between mitotic index and Eg5 gene expression level in breast tumors. Recent researches also showed that Eg5 has been increasingly implicated in tumorigenesis ${ }^{[20-22]}$. Tang et al. ${ }^{[23]}$ showed that Eg5 was expressed in all cases of head and neck squamous cell carcinoma, and the level of Eg5 expression was correlated with the proliferation activity of cancer cells. A series of investigations on the possible mechanism of $\mathrm{Eg} 5$ in cancers have been studied. Research showed that intracellular Eg5 is closely related to the MAPK cascade and that the expression of Eg5 in tumor derived cell lines leads to the induction of resistance to monastrol in cells ${ }^{[24-25]}$. Several inhibitors for Eg5 have been reported, such as K858, S-trityl-L-cysteine and pyrazolopyrimidine derivates ${ }^{[26-28]}$. These experimental data show that Eg5 is oncogenic and may be a therapeutic target for discovering novel cancer treatments.

However, little work on the prognostic significance of Eg5 in human ovarian cancer has been conducted. Based on the information obtained from this study, a substantial relationship was demonstrated between Eg5 expression and poor overall survival in patients with ovarian cancer by univariate and multivariate survival analyses $(P<0.05)$. Therefore, Eg5 could predict an unfavorable prognosis as an independent marker of EOC. This is in accordance with previous studies which identified that a low overall survival rate is correlated with a high expression of Eg5 in certain cancers, for example, renal cell carcinoma and hepatocellular carcinoma ${ }^{[29-30]}$.

Interestingly, studies have shown a significant nuclear expression of Eg5 in prostate cancer ${ }^{[31]}$. In comparison, we mainly observed cytoplasmic expression of Eg5 in EOC samples, while the nuclear expression of Eg 5 was ruled out by statistical analysis. This may be due to the differences in the tumor types and antibodies used. In future research, enrolling larger samples or with the ability to perform in vitro and in vivo experiments are necessary to investigate the role of Eg5 in EOC tumor biology.

Overall, this study analyzed the expression of Eg5 in ovarian cancer quantitatively. The data found that Eg5 was highly expressed in EOC, suggesting that high Eg5 expression is related to poor overall survival. Eg5 is an independent prognostic factor of EOC. More studies are necessary to verify the function of $\mathrm{Eg} 5$ as an oncogene in EOC, in order to deliver promising applications in its diagnosis and treatment.

\section{Acknowledgments}

This study was supported by grants from the Frontier and Key Technologies Research Projects (MS12017013-3) and the Nantong New Clinical Diagnosis and Treatment Technology Project (MS12017013-1).

\section{References}

[1] Roane BM, Arend RC, Birrer MJ. Review: targeting the transforming growth Factor-Beta pathway in ovarian cancer[J]. Cancers (Basel), 2019, 11(5): E668

[2] Lawrenson K, Song FJ, Hazelett DJ, et al. Genome-wide association studies identify susceptibility loci for epithelial ovarian cancer in East Asian women[J]. Gynecol Oncol, 2019, 153(2): 343-355

[3] Gourley C, Bookman MA. Evolving concepts in the management of newly diagnosed epithelial ovarian cancer[J]. J Clin Oncol, 2019, 37(27): 2386-2397

[4] Tong L, Wang Y, Ao Y, et al. CREBl induced lncRNA HAS2-AS1 promotes epithelial ovarian cancer proliferation and invasion via the miR-466/RUNX2 axis[J]. Biomed Pharmaco, 2019 ,115:108891.

[5] Yuan SJ, Qiao TK, Qiang JW, et al. Dynamic contrast enhanced-magnetic resonance imaging for the early evaluation of the response to docetaxel in rats with epithelial ovarian cancer[J]. Oncol Rep, 2019, 41(6): 3335-3346

[6] Seraj S, Lee J, Ahn HJ. Systemic delivery of Eg5 shRNAexpressing plasmids using PEGylated DC-Chol/ DOPE cationic liposome: Long-term silencing and anticancer effects in vivo[J]. Biochem Pharmacol, 2019, 166(192/202): 192-202

[7] Jungwirth G, Yu T, Moustafa M, et al. Identification of KIF11 as a novel target in meningioma[J]. Cancers, 2019, 11(4): E545.

[8] Novak M, Polak B, Simunić J, et al. The mitotic spindle is chiral due to torques within microtubule bundles[J]. Nat Commun, 2018, 9(1): 3571

[9] Valentine MT, Gilbert SP. To step or not to step?How biochemistry and mechanics influence processivity in Kinesin and Eg5[J].Curr Opin Cell Biol,2007,19(1):7581

[10] Lu M, Zhu H, Wang X, et al. The prognostic role of Eg5 expression in laryngeal squamous cell carcinoma[J]. $\mathrm{Pa}-$ thology, 2016, 48(3): 214-218

[11] Lee DJ, Kessel E, Edinger D, et al. Dual antitumoral potency of EG5 siRNA nanoplexes armed with cytotoxic bifunctional glutamyl-methotrexate targeting ligand[J]. Biomaterials, 2016, 77(98/110): 98-110

[12] Liu L, Liu X, Mare M, et al. Overexpression of Eg5 cor- 
relates with high grade astrocytic neoplasm[J]. J Neurooncol, 2016, 126(1): 77-80

[13] Castillo A, Morse HC, Godfrey VL, et al. Overexpression of Eg5 causes genomic instability and tumor formation in mice[J]. Cancer Res, 2007, 67(21): 10138-10147

[14] Xing ND, Ding ST, Saito R, et al. A potent chemotherapeutic strategy in prostate cancer: S-(methoxytrityl)-Lcysteine, a novel Eg5 inhibitor[J]. Asian J Androl, 2011, 13(2): 236-241

[15] Giantulli S, De IF, Taglieri L, et al. Growth arrest and apoptosis induced by kinesin Eg5 inhibitor K858 and by its 1,3,4-thiadiazoline analogue in tumor cells[J]. Anticancer Drugs, 2018, 29(7): 674-681

[16] De Iuliis F, Taglieri L, Salerno G, et al. The kinesin Eg5 inhibitor K858 induces apoptosis but also survivinrelated chemoresistance in breast cancer cells[J]. Invest New Drugs, 2016, 34(4): 399-406

[17] Chen GY, Cleary JM, Asenjo AB, et al. Kinesin-5 promotes microtubule nucleation and assembly by stabilizing a Lattice-Competent conformation of tubulin[J]. Curr Biol, 2019, 29(14): 2259-2269.e4

[18] Hayashi N, Koller E, Fazli L, et al. Effects of Eg5 knockdown on human prostate cancer xenograft growth and chemosensitivity[J]. Prostate, 2008, 68(12): 1283-1295

[19] Marcus AI, Peters U, Thomas SL, et al. Mitotic kinesin inhibitors induce mitotic arrest and cell death in Taxolresistant and -sensitive cancer cells[J]. J Biol Chem, 2005, 280(12): 11569-11577

[20] Sherin L, Farwa S, Sohail A, et al. Cancer drug therapy and stochastic modeling of "nano-motors". International journal of nanomedicine, 2018, 13(6429-40).

[21] Truebenbach I, Zhang W, Wang Y, et al. Co-delivery of pretubulysin and siEG5 to EGFR overexpressing carcinoma cells[J].Int J Pharm, 2019, 569(118570): 118570

[22] Ogunwa TH, Laudadio E, Galeazzi R, et al. Insights into the Molecular Mechanisms of Eg5 Inhibition by
(+)-Morelloflavone[J].Pharmaceuticals (Basel), 2019, 12(2): 58

[23] Tang P, Siu LL, Chen EX, et al. Phase II study of ispinesib in recurrent or metastatic squamous cell carcinoma of the head and neck[J]. Invest New Drugs, 2008, 26(3): 257-264

[24] Liang YJ, Yang WX. Kinesins in MAPK cascade: How kinesin motors are involved in the MAPK pathway?[J]. Gene, 2019, 684(1/9): 1-9

[25] Tcherniuk S, Van Lis R, Kozielski F, et al. Mutations in the human kinesin Eg5 that confer resistance to monastrol and S-trityl-L-cysteine in tumor derived cell lines[J]. Biochem Pharmacol, 2010, 79(6): 864-872

[26] Taglieri L, Rubinacci G, Giuffrida A, et al. The kinesin Eg5 inhibitor K858 induces apoptosis and reverses the malignant invasive phenotype in human glioblastoma cells[J]. Invest New Drugs, 2018, 36(1): 28-35

[27] $\mathrm{Wu} \mathrm{W}$, Jingbo S, Xu W, et al. S-trityl-L-cysteine, a novel Eg5 inhibitor, is a potent chemotherapeutic strategy in neuroblastoma[J]. Oncol Lett, 2018, 16(1): 1023-1030

[28] Muthuraja P, Veeramani V, Prakash S, et al. Structureactivity relationship of pyrazolo pyrimidine derivatives as inhibitors of mitotic kinesin Eg5 and anticancer agents[J]. Bioorg Chem, 2019, 84(493/504): 493-504

[29] Sun D, Lu J, Ding K, et al. The expression of Eg5 predicts a poor outcome for patients with renal cell carcinoma[J]. Med Oncol, 2013, 30(1): 476

[30] Liu C, Zhou N, Li J, et al. Eg5 overexpression is predictive of poor prognosis in hepatocellular carcinoma patients[J]. Dis Markers, 2017(2176460): 2176460

[31] Jin Q, Dai Y, Wang Y, et al. High kinesin family member 11 expression predicts poor prognosis in patients with clear cell renal cell carcinoma[J]. J Clin Pathol,2019,72(5):354-362.

(Received 9 October 2019, Revised 2 November 2019, Accepted 4 December 2019) 